\title{
FASTER AND FASTER CONVERGENT SERIES FOR $\zeta(3)$
}

\author{
TEWOdRos AmdeBERHAN \\ Department of Mathematics, Temple University, Philadelphia PA 19122, USA \\ tewodros@euclid.math.temple.edu
}

Submitted: April 8, 1996. Accepted: April 15, 1996

ABstract. Using WZ pairs we present accelerated series for computing $\zeta(3)$

AMS Subject Classification: Primary 05A

Alf van der Poorten $[\mathrm{P}]$ gave a delightful account of Apéry's proof $[\mathrm{A}]$ of the irrationality of $\zeta(3)$. Using WZ forms, that came from [WZ1], Doron Zeilberger [Z] embedded it in a conceptual framework.

We recall [Z] that a discrete function $\mathrm{A}(\mathrm{n}, \mathrm{k})$ is called Hypergeometric (or Closed Form $(\mathrm{CF})$ ) in two variables when the ratios $A(n+1, k) / A(n, k)$ and $A(n, k+1) / A(n, k)$ are both rational functions. A pair $(\mathrm{F}, \mathrm{G})$ of $\mathrm{CF}$ functions is a WZ pair if $F(n+1, k)-F(n, k)=G(n, k+1)-G(n, k)$. In this paper, after choosing a particular $\mathrm{F}$ (where its companion $\mathrm{G}$ is then produced by the amazing Maple package EKHAD accompanying [PWZ]), we will give a list of accelerated series calculating $\zeta(3)$. Our choice of $\mathrm{F}$ is

$$
F(n, k)=\frac{(-1)^{k} k !^{2}(s n-k-1) !}{(s n+k+1) !(k+1)}
$$

where $\mathrm{s}$ may take the values $\mathrm{s}=1,2,3, \ldots[\mathrm{AZ}]$ (the section pertaining to this can be found in http://www.math.temple.edu/ ${ }^{\sim}$ tewodros). In order to arrive at the desired series we apply the following result:

Theorem: ([Z], Theorem 7, p.596) For any WZ pair (F,G)

$$
\sum_{n=0}^{\infty} G(n, 0)=\sum_{n=1}^{\infty}(F(n, n-1)+G(n-1, n-1))
$$

whenever either side converges.

The case $\mathrm{s}=1$ is Apéry's celeberated sum $[\mathrm{P}]$ (see also $[\mathrm{Z}]$ ):

$$
\zeta(3)=\frac{5}{2} \sum_{n=1}^{\infty}(-1)^{n-1} \frac{1}{\left(\begin{array}{c}
2 n \\
n
\end{array}\right) n^{3}}
$$

where the corresponding $\mathrm{G}$ is

$$
G(n, k)=\frac{2(-1)^{k} k !^{2}(n-k) !}{(n+k+1) !(n+1)^{2}}
$$

Typeset by $\mathcal{A} \mathcal{M} \mathcal{S}-\mathrm{T}_{\mathrm{E}} \mathrm{X}$ 
For $\mathrm{s}=2$ we obtain

$$
\zeta(3)=\frac{1}{4} \sum_{n=1}^{\infty}(-1)^{n-1} \frac{56 n^{2}-32 n+5}{(2 n-1)^{2}} \frac{1}{\left(\begin{array}{c}
3 n \\
n
\end{array}\right)\left(\begin{array}{c}
2 n \\
n
\end{array}\right) n^{3}}
$$

where $\mathrm{G}$ is

$$
G(n, k)=\frac{(-1)^{k} k !^{2}(2 n-k) !(3+4 n)\left(4 n^{2}+6 n+k+3\right)}{2(2 n+k+2) !(n+1)^{2}(2 n+1)^{2}} .
$$

For $\mathrm{s}=3$ we have

$$
\zeta(3)=\sum_{n=0}^{\infty} \frac{(-1)^{n}}{72\left(\begin{array}{c}
4 n \\
n
\end{array}\right)\left(\begin{array}{c}
3 n \\
n
\end{array}\right)}\left\{\frac{6120 n+5265 n^{4}+13761 n^{2}+13878 n^{3}+1040}{(4 n+1)(4 n+3)(n+1)(3 n+1)^{2}(3 n+2)^{2}}\right\},
$$

and so on.

\section{REFERENCES}

[A] R. Apéry, Irrationalitè de $\zeta(2)$ et $\zeta(3)$, Asterisque 61 (1979), 11-13.

[AZ] T. Amdeberhan, D. Zeilberger, WZ-Magic, in preparation.

[PWZ] M. Petkovšek, H.S. Wilf, D.Zeilberger, " $A=B$ ", A.K. Peters Ltd., 1996.

The package EKHAD is available by the www at http://www.math.temple.edu/ zeilberg/programs.html

[P] A. van der Poorten, A proof that Euler missed ..., Apéry's proof of the irrationality of $\zeta(3)$, Math. Intel. 1 (1979), 195-203.

[WZ1] H.S. Wilf, D. Zeilberger, Rational functions certify combinatorial identities, Jour. Amer. Math. Soc. 3 (1990), 147-158.

[Z] D. Zeilberger, Closed Form (pun intended!), Contemporary Mathematics 143 (1993), 579-607.. 